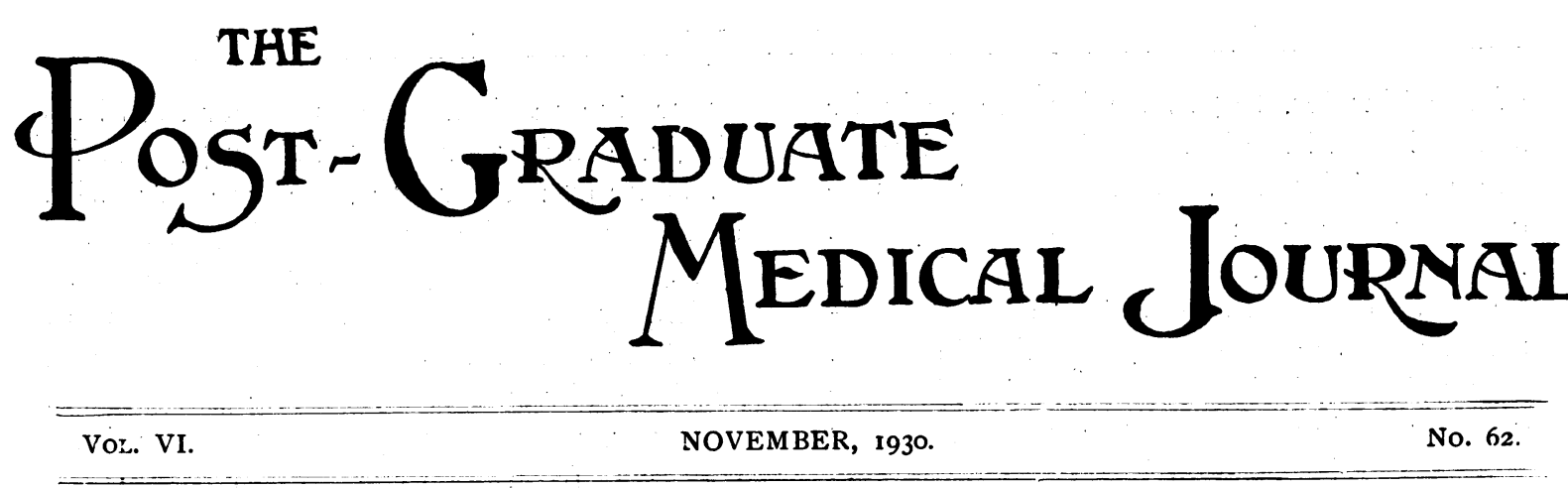

\title{
CONTENTS
}

MALIGNANT DISEASE OF THE PROSTATE AND BLADDER $\ldots$ PAGE

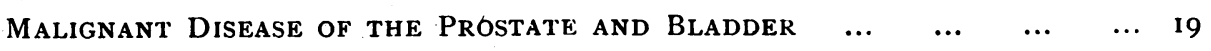

By H. L. Attwater, M.A., M.Chir.Cantab., F.R.C.S.Eng.

$\begin{array}{llllllllllll}\text { OBSTRUCTION OF THE COLON } & \ldots & \ldots & \ldots & \ldots & \ldots & \ldots & \ldots & \ldots & 24\end{array}$

By W. B. GABRIEL, M.S., F.R.C.S.

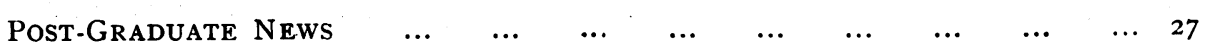

$\begin{array}{llllllllllllll}\text { NOTICES } & \ldots & \ldots & \ldots & \ldots & \ldots & \ldots & \ldots & \ldots & \ldots & \ldots & \ldots & \ldots & \text { iii }\end{array}$

Fellowship of Medicine and Post-Graduate Medical Association.-

$\begin{array}{llllllllllll}\text { SPECIAL COURSES } & \ldots & \ldots & \ldots & \ldots & \ldots & \ldots & \ldots & \ldots & \ldots & \text { ii }\end{array}$

\section{MALIGNANT DISEASE OF THE PROSTATE AND BLADDER.}

\author{
By H. L. ATTWATER, \\ M.A., M.CHIR.CANTAB., F.R.C.S.ENG.,
}

Honorary Assistant Surgeon, All Saint's Hospital.

\section{(Continued from p. 10.)}

The appearance of a nodular mass, often ulcerated, is generally due to the presence of a carcinoma of the bladder wall. They are not nearly so common as the preceding forms but, as a rule, are easy to diagnose.

We will now consider the treatment of these diseases and will first discuss the methods of dealing with malignant disease of the prostate. Many cases, when they come for help, are so far advanced that, whilst radical removal is out of the question the distress is so great that something must be done for the relief of the unfortunate sufferer. Happily, the symptoms are often due to obstruction to the outflow of urine which can easily be remedied by the establishment of a permanent suprapubic fistula which provides relief from obstruction. A large number of such cases, when a suitable suprapubic apparatus has been fitted, become greatly benefited and are freed from all but the relatively slight discomfort caused by the apparatus. Painful spasms of the urethra often occur and may persist after the establishment of drainage. This is, however, usually met by attention to the cystitis by means of suitable irrigations, by the giving of urinary sedatives, $c f$., hyoscyamus, $\&$ c., and by attention to the diet and regular regime of the patient, so that the urine may be kept in as non-irritating a condition as possible.

We now turn to the question of radical removal of malignant growths of the prostate. A great deal of work has been done on this subject, notably in America, by Young. He has devised a technique for the complete removal of the prostate and adjacent parts 
of the bladder, together with the vesiculæ seminales and the infiltrated terminations of the vasa deferentia. When one comes to consider the results of these operations, there appears little doubt that the difficulty lies not so much in the nature of the operation as in the choosing of suitable cases. Early diagnosis is essential, and unless we get our patient before the neoplasm has encroached into the pelvic planes and has commenced to anchor the proslate to adjacent structures, we shall not be able to extirpate the growth completely by operation, and recurrence of the trouble at an early date is then only to be expected.

Young's perineal operation and other methods, many of which are adaptations or modifications of his, are excellent procedures provided that the case is seen sufficiently early, but if there is any doubt as to the suitability of any particular case, it is probably best to leave it alone from the point of view of radical excision. A complete survey of every case must be made, not only as to the local condition of the prostate but also as to the age of the patient, the state of renal function, the presence or absence of glands, evidence of metastasis, and of intercurrent disease. The presence of any adverse factor must necessarily modify our decisions in any particular case.

During the past few years attention has been directed with increasing strength and frequency to the question of the action of certain forms of electro-magnetic radiation on the growth of malignant tissues. The number of published cases in which benefit has resulted is considerable, and it would seem that still better results may be hoped for as improvements are discovered. There are two chief methods of applying this treatment; the one is by means of what has been called "deep X-ray therapy," and the other by means of the radiations derived from radium.

With regard to the treatment of prostatic cases by means of deep X-rays, it would appear that it is better to apply the rays in successive moderate doses rather than in a single, or a few, prolonged sittings. As far $\stackrel{\mathbb{Q}}{\varrho}$ as is known at present, we can say with certainty that the progress of growths has $\stackrel{\overrightarrow{3}}{\overrightarrow{3}}$ been checked and the lives of patients? definitely prolonged in many cases of 흠 prostatic cancer which have been subjected $\vec{\sigma}$ to deep X-ray therapy. The results are, $\stackrel{\varrho}{\Omega}$ however, not uniform, and we are a long way yet from being able to express a final opinion on the subject.

Much the saine may be said with regard to the application of radium. Its rays appear to have a definite selective action on cancer cells and, as a rule, application of the o rays causes immediate benefit with respect $\overrightarrow{0}$ to symptoms. As to whether any case has 음 been completely cured is doubtful, though $\rightarrow$ many are on record in whom the disease $z$ has remained quiescent, has apparently disappeared, and the life of the patient has been prolonged for ten or more years after the application of the radio-active materiap

The treatment may have certain harmf un effects which should not be overlooked. The action of the rays causes congestion and a great proliferation of fibrous tissue which, by its contraction, may cause subsequent interference with vital organs such as the ends of the ureters, where they enter the bladder, causing back pressure and renal failure. During the application, if great care is not exercised, inflammation of the adjacent mucous membranes may be set up with great subsequent pain and suffering; the latter difficulty is, however, avoided by careful technique. The rays appear to have a selective action on cancer cells, and it is noteworthy that the more malignant the type of growth the greater destructive effect the rays appear to have on it.

There are several methods of applying radium to prostatic cancer. It can be used by means of suitable applicators, so that the rays will pass through healthy tissues and radiate the growth in their passage. When used in this way a quantity of radium salt, in a container suitably screened to prevent 
the action of the $a$ and $\beta$ rays, is placed on the surface of the skin in the region of the prostate. In doing this the amount of radiation which it is desired to give the prostate is calculated, and also the amount which will not cause more than a mild reaction of the skin. The total application necessary to deal with the neoplasm must then be divided between different skin areas and given in several sittings, so that whilst the prostate receives its full dose, the time to which any individual area of the skin is subjected to the action of the rays will not cause more than a mild reaction. The total dose may therefore have to be divided into two, three, four, or more applications to different skin areas surrounding the prostate.

Application may also be made by a suitable radium container passed into the rectum, or to the trigone of the bladder, by means of a mechanical cystoscope. Both these methods are, however, limited because of the liability to set up acute inflaımmation of the mucosa of the rectum or bladder.

A more efficacious method at the present time is to apply the salt in needles. These are small cylindrical tubes of such material and thickness as will provide adequate screening. The radium is placed in the interior of the cylinder, one end of which is sharp and the other end of which possesses an eye to which a silk thread can be attached. When needles are to be implanted the prostate is exposed surgically, either by the perineal or the suprapubic routes, and a number of needles, carefully spaced, are inserted into the substance of the carcinomatous organ, the silk threads being allowed to come out through the external wound. In this manner the whole gland can be brought under the influence of the radiation at short range. The action of the rays is allowed to continue until the proper dose has been given, when the threads are pulled on and the needles withdrawn. Great care must be exercised both to estimate the dose and to space the needles correctly, so that whilst no area is in danger of receiving too much of the rays, yet at the same time areas in between the situations of the needles are not allowed to escape being radiated.

At the present time the above method of application is probably the most efficient, but recently the implantation of radon "seeds" has been adopted and may supersede the former method, when the technique has been further explored and perfected. In this method a quantity of radium is made to give off a gas, known as radium emanation or radon. This gas is passed into suitable tubes, capillary in size, and gives off active $\boldsymbol{\gamma}$ radiations. Only a small amount of gas is given off, relative to the weight, of radium used, but the supply is maintained during the life of radium itself, so that for all practical purposes it is inexhaustible. The capillary tubes containing the radon are cut into short lengths or "seeds," and the latter can be implanted into the substance of a growth in the same manner as the radium needles. There is, however, this great difference that the action of the radon comes? to an end after about four days. Sufficient "seeds" must, therefore, be implanted to give the required radiation in that interval. The advantages are that the radium itself is not used and remains in the laboratory as a permanent supply of radium emanation, and the little "seeds" are so small that they can safely be left in the tissues and need not be removed.

From the above it will be seen that the technique of treating a case with radium is highly important. Not only must the radioactive substance be placed accurately in relation to the growth, but a careful estimate must be made of the dose, both as to the amount of radium and of the time for which it acts. Also screening necessary to prevent the action of harmful rays must be provided. If these points are not attended to disastrous sloughing may occur with highly distressing results. When the substance is properly applied there is no doubt that many cases respond to this mode of treat. 
ment in an almost miraculous manner. Not only is the distress of the patient alleviated and health restored, but frequently life has been prolonged for many years. This has occurred to such.an extent in some instances that actual cure may perhaps be hoped for along these lines.

As at present neither surgery nor radium, when used alone, are entirely satisfactory in the treatment of cancer of the prostate, let us now consider the possibility of combining these methods of attacking malignant growths. Two courses suggest themselves : first, to expose the growth to radiation before operation, then to excise the neoplasm, with or without further application of the rays to the site of the disease; secondly, to excise the growth and then to subject the wound to efficient radiation with a view to stamping out any remuants of neoplasm which may have been left behind at the operation.

There is an important objection to the first method, which is that the inflammatory reaction set up by the preliminary application of the rays and the subsequent fibrosis often make the operation of excision one of great difficulty. The removal of the tumour may be " ragged," with great liability to leave cancer-infected fragments behind. The second method appears to be more feasible ; the mass of the disease is removed, leaving less for the rays to deal with, and consequently there is more hope that any remaining malignant elements may be successfully radiated and destroyed, if the radium is applied along the directions in which the growth is liable to extend.

More and more cases are being recorded in which either the growth has been excised and the field radiated or, when excision has been impossible, in which the neoplasm has bean subjected to the action of the rays alone. In many of these the growth has shrivelled and in some would seem to have disappeared.

Let us now consider the similar treatment of malignant growths of the bladder. When only palliative procedures are possible there is little doubt that radium is extremely useful. Personally, I believe that radium should be used in all such cases, because of the possibility of obtaining by its use far longer prolongation of life than by any other means. Simple cystotomy, with or without the application of diathermy to destroy the mass of the growth, is useful in giving great relief but, if radium is employed, results are often obtained which go considerably further ; bleeding and pain are checked, and the comfort of the patient is often increased enormously.

The subject is complicated in the case of the bladder by the existence of more than one type of malignant growth. Malignant papillomata of recent origin, in which the carcinomatous changes are confined to the tips of the papillæ, may sometimes be dealt with quite satisfactorily by means of diathermic destruction alone. If, however, the cancer involves the pedicle and is invading the wall of the bladder, diathermy alone wilf not suffice. Such simple proceedings at cystotomy, with or without the use of dia? thermy, only give relief by putting the bladder at rest, and may even fail to do this if the organ has become irritable. Painful spasms may persist and make the life of the patient miserable, especially if, as occasionally happens, he is nervous and intolerant to treatment.

When the growth is known to be early and is favourably situated there is no doubt that radical operation for removal still holds out the best hope of success. The procedures to be adopted fall under two heads, either total cystectomy, or the partial excision of the growth-bearing portion of the vesical wall.

Total cystectomy has undoubtedly had its successes, and in the hands of a few surgeons patients appear to have been saved from a cancerous death, life being prolonged in comparative comfort for many years. The procedure is, however, a drastic one and has a high mortality ; the ureters must be transplanted and the patient may have to wear a 
ureteric apparatus for the rest of his life with all its attendant discomforts. Moreover, the operation for the transplantation of the ureters is still not always satisfactory, and a considerable number of these cases die of ascending renal sepsis directly caused by the removal of the ureters from their natural positions. It would appear that the operation has only a limited application and should only be done when the indications for it are clearly defined.

The operation of choice would appear to be the excision of the portion of the wall of the bladder which bears the growth, together with a sufficient margin of the surrounding vesical wall which will make certain of being quite clear of malignant extensions. This operation, conducted on modern lines, in suitable cases holds out a much more favourable hope of permanent benefit and undoubtedly has frequently cured the disease. As much as one half, or a little more, of the bladder may be removed and the remainder will rapidly accommodate itself, producing an organ with good capacity and normal action.

The site of the growth plays an important part with regard to prognosis. The most favourable site, but unhappily not a very common one, is when the neoplasm is situated on the vertex or high up on the lateral vesical wall. The nearer to the base the growth is placed the less favourable it is for removal, though a small growth situated by one ureteric orifice may often be removed successfully, the end of the affected ureter being cut across and reimplanted into the bladder.

Some authorities are so strongly of opinion that cystectomy, total or partial, is the right course to pursue in these cases that they are definitely opposed to the ray treatment. Personally, I believe that when the growth in the bladder is early and can be removed with reasonable ease, this is the right course to adopt. If, however, the operation is likely to be difficult, owing to the growth being situated on the base of the bladder, being too extensive, or involving both ureteric orifices, then it would seem that radium should be used.

With regard to bladder growths it appears that radium, as opposed to X-rays, holds out the best hope of attacking the disease successfully. A great deal has been said and written on the two methods, but as yet no very reliable or final statistics have been produced; but my impression is that radium is the more useful agent of the two. It is clear that, whereas in the case of the prostate both radium and $\mathrm{X}$-rays have shown their value and many successful cases have been recorded, in the case of the bladder there is not quite the same consensus of favourable opinion. This may be due to the inflammatory reaction which is so easily set up in the bladder and which vitiates against the best results.

As to the method of applying radium to the bladder, this is practically the same as in the case of the prostate, either from a distance by means of an applicator on the skin, byy means of a cystoscopic apparatus which enables the radio-active substance to be placed in close contact with the growth within the bladder, or by the implantation of needles containing radium, or radon " seeds," placed in position through a cystotomy wound.

From the rather less favourable results which occur with radiating growths of the bladder, it is clear that attempts to avoid surgical removal of a growth in operable cases are less to be countenanced than in the case of the prostate, and that radium should not be relied on alone unless it is the only method which is applicable. Early treatment of a case of vesical cancer is essential, and it is of paramount importance that warning symptorns, such as a slight transient hæmaturia, should be investigated at once. In such cases, when favourable and obtained early, the growth should be excised, and the site of operation should be exposed to the action of radium at the time of excision, and perhaps the whole area may be 
given a course of "deep X-ray therapy" for some weeks during convalescence and after.

In the present state of our knowledge I feel that it is doubtful policy to rely too much on any one method of treatment for these cases, but that we should remove the growth as cleanly and as widely as possible, and then subject the site of operation to radio-activity in order to stamp out any elements of cancer which may remain. Finally, we must pay every attention and concentrate our efforts towards improving technique and correlating results.

With regard to the results of treatment of malignant disease of the prostate and bladder, we must admit that the outlook is still far from bright and that a large number of these people do not survive more than a year or so after the onset of symptoms. The great difficulty is the insidious nature of the onset of the trouble, which makes it essential that we should be on the outlook for early symptoms, so that we may be able to proceed with more hope of a successful issue, and not, as is too often the case, meet our patient when he is beyond hope of anything but the possibility of making him comfortable and perhaps of adding a few months to his existence.

Little remains; we have in surgery and radiation treatment two powerful weapons which, if given a chance, will help us greatly, but too much must not be expected of them at present. The disease is a desperate one and the results of treatment are still imperfect. There has, however, been some improvement, and it is encouraging to find that without doubt in many cases we have a method which will often allay symptoms, cause shrinkage of the growth, and prolong life.

\section{OBSTRUCTION OF THE COLON.}

\author{
$B y$ W. B. GABRIEL,
}

M.S., F.R.C.S.,

Surgeon to the Royal Northern Hospital, Senior Assistant Surgeon to St. Mark's Hospital for Diseases of the Rectum.

IT is the common experience among surgeons that resection of the colon is a tricky and dangerous operation. The factors which contribute to the high mortality of resection and anastomosis of the colon are as follows :-

(I) The patients are often advanced in age, with poor healing powers.

(2) A state of subacute obstruction often exists which it is difficult to overcome even after careful bowel preparation.

(3) The contents of the colon are extremely infective and the risk of peritonitis is much greater than after resection of any other part of the gastro-intestinal tract.

(4) The technical difficulties in rnaking thoroughly sound anastomosis, whether lateral or end-to-end, are often very great; there may be great disproportion in size between the bowel lumen above and below the site of obstruction; atrophy or hypertrophy of the bowel wall may make suturing difficult, and the overloading with fat and enlarged appendices epiploicæ which is so commonly met with make it difficult to insert a uniformly firm line of peritoneal sutures. The blood supply to the upper and lower ends has to be carefully considered on anatomical lines if a $\mathrm{V}$-shaped portion of meso-colon is to be excised, and excessive zeal in placing sutures has to be avoided for fear of damaging the vascularity of the anastomosis. Even if a cæcostomy is done at the conclusion of the operation the risk of leakage at the junction is not entirely prevented. Other points calling for anxious consideration by the surgeon are whether local drainage should be arranged for at the side of the anastomosis and the decision as 